\title{
金属空气电池阴极氧还原催化剂研究进展
}

\author{
王瀛* 张丽敏胡天军 \\ (山西师范大学化学与材料科学学院 临汾 041004)
}

\begin{abstract}
摘要 随着能源危机加剧和生态环境恶化, 可持续发展能源受到更大的重视. 金属空气电池作为一种绿色能源是具有 很大发展潜力的新一代电池. 与传统电池相比, 此类电池有着更高的理论能量密度, 尤其是锂空电池, 能量密度可达 $3505 \mathrm{Wh} / \mathrm{kg}$, 然而阴极缓慢的氧还原反应成为制约其发展的关键因素之一. 在简要介绍氧还原反应机理基础上, 着重 介绍了近年来氧还原催化剂如贵金属及其合金、过渡金属氧化物/硫化物、功能化碳材料和金属氮化物的研究进展, 并 根据目前所存在问题指出未来研究方向, 包括深入研究氧还原反应机理, 明确催化剂活性位; 研究催化剂结构等对催 化活性的影响, 优化制备条件, 以提高催化活性和稳定性; 根据氧还原机理设计开发新型氧还原催化剂.
\end{abstract}

关键词 金属空气电池; 氧还原反应; 催化剂; 电解液; 反应机理

\section{Progress in Oxygen Reduction Reaction Electrocatalysts for Metal-Air Batteries}

\author{
Wang, Ying* Zhang, Limin Hu, Tianjun \\ (School of Chemistry and Material Science, Shanxi Normal University, Linfen 041004)
}

\begin{abstract}
With the intensification of the global energy crisis and the deterioration of ecological environment, the exploitation and utilization of sustainable energy have gained more attention. Metal-air battery as a kind of high-performance green energy may become one of the most promising next-generation battery technologies. Compared to conventional storage batteries such as $\mathrm{Zn}-\mathrm{Mn}$ and lead-acid batteries, metal-air battery has higher theoretical energy density, especially Li-air battery with an extremely high theoretical density $3505 \mathrm{Wh} / \mathrm{kg}$. Such high energy density is due to the fact that oxygen is not stored in the cell. Other advantages include stable potential, low cost and environmental friendship. However, there are many important factors that limit its commercial application. Among them, a critical issue is the sluggish kinetics of cathodic oxygen reduction reaction (ORR), so it is necessary to develop ORR catalytic materials for enhancing the kinetics. Recently, there are many researches about ORR catalysts. In addition to a brief introduction of the reaction mechanism of ORR, the paper introduced the current research progress of four groups of cathodic catalysts including noble metal and its alloys, transition-metal oxides/sulfides, functional carbon materials and metal nitrides. However, there are still many problems, such as the lack of fundamental mechanistic study, high cost of Pt-based catalyst, uncertain active site of functional carbon materials and low activity for non-noble metal catalysts. In summary, great efforts should be needed. Based on this, the authors pointed out the development direction for ORR catalysts. The future research direction of cathodic catalysts would include: (1) researching the elusive oxygen reaction mechanism and defining the active sites, (2) studying the effect of physical structure parameters (e.g., structure, morphology, size) on the activity and optimizing synthesis conditions of catalysts to obtain better activity and stability, (3) developing novel efficient and inexpensive catalysts in according to the oxygen reaction mechanism.

Keywords metal-air batteries; oxygen reduction reaction; catalyst; electrolyte; reaction mechanism
\end{abstract}

\section{1 引言}

随着煤、石油、天然气等不可再生能源的逐渐枯竭 及全球气候变化, 可持续发展能源的开发与应用成为未 来人类社会发展所面临的重要问题之一. 金属空气电池 作为一种绿色能源, 具有无毒、无污染、放电电压平稳、 高比能量、储存寿命长、价格相对较低等优点. 此类电 池是以金属(锌、锂、铝等)和氧气为燃料将化学能转变 为电能, 既有丰富的廉价资源, 又可再生利用, 而且比 氢燃料电池结构简单, 是很有应用前景的新能源 ${ }^{[1]}$. 与
现有的铅酸、镍氢等电池相比, 金属空气电池具有更高 的能量密度和比能量, 尤其是锂空电池, 其能量密度理 论值可达 $3505 \mathrm{Wh} / \mathrm{kg}^{[2]}$, 远高于锂离子电池 ${ }^{[3 \sim 5]}$. 目前, 金属空气电池的研究已取得较大进展, 然而电池阴极缓 慢的氧还原反应制约了整个电池的性能，成为其商业化 应用道路上的一个障碍. 为进一步提高电池性能, 国内 外学者对氧还原催化剂进行了广泛研究 ${ }^{[6 \sim 8]}$. 本文结合 金属空气电池工作原理, 较为系统地综述了近年来氧还 原催化剂的研究进展, 并对其发展趋势进行了探讨.

\footnotetext{
*E-mail: wangyme@163.com; zhanglm@sxnu.edu.cn

Received November 16, 2014; published January 23, 2015.

Project supported by Natural Science Foundation of Shanxi Normal University (No. ZR1402).

项目受山西师范大学自然科学基金(No. ZR1402)资助.
} 


\section{2 氧还原反应机理}

由于氧还原反应发生在气、液、固三相间, 因此这 样一个复杂的环境也就造成其反应机理是非常复杂的. 根据反应环境的不同, 氧还原反应机理大体可分为两 种: 水系电解液体系和非水电解液体系.

\section{1 水系电解液体系}

由于大多数金属在酸性电解液中会发生严重的腐 蚀或析氢现象, 同时在碱性溶液中氧还原反应较快且过 电位较低, 因此对于金属空气电池, 电解液一般选用碱 性溶液为电解液，其放电反应式如下:

$$
\begin{aligned}
& \text { 负极: } \mathrm{M} \rightarrow \mathrm{Mn}^{+}+n \mathrm{e}^{-}(\mathrm{M} \text { 为金属 }) \\
& \text { 正极: } \mathrm{O}_{2}+2 \mathrm{H}_{2} \mathrm{O}+4 \mathrm{e}^{-} \rightarrow 4 \mathrm{OH}^{-} \\
& \text {总反应: } 4 \mathrm{M}+n \mathrm{O}_{2}+2 n \mathrm{H}_{2} \mathrm{O} \rightarrow 4 \mathrm{M}(\mathrm{OH})_{n}
\end{aligned}
$$

目前普遍认为, 氧还原反应一般有两种途径: 四电 子转移和二电子转移. 具体反应机理如下.

(1)四电子转移过程:

$\mathrm{O}_{2}+2 \mathrm{H}_{2} \mathrm{O}+4 \mathrm{e}^{-} \rightarrow 4 \mathrm{OH}^{-}$

(2)二电子转移过程:

$$
\begin{aligned}
& \mathrm{O}_{2}+\mathrm{H}_{2} \mathrm{O}+2 \mathrm{e}^{-} \rightarrow \mathrm{HO}_{2}^{-}+\mathrm{OH}^{-} \\
& \mathrm{HO}_{2}^{-}+\mathrm{H}_{2} \mathrm{O}+2 \mathrm{e}^{-} \rightarrow 3 \mathrm{OH}^{-} \\
& 2 \mathrm{HO}_{2}^{-} \rightarrow 2 \mathrm{OH}^{-}+\mathrm{O}_{2}
\end{aligned}
$$

当氧还原按四电子途径反应时, 氧分子被还原为氢 氧根离子; 当按二电子途径反应时, 产物中除有氢氧根 离子外, 还会有大量过氧化物产生. 过氧化物一方面降 低了氧还原反应效率, 同时它还具有强氧化作用, 损坏 电池隔膜而影响循环寿命, 因此对于水系电解液而言, 四电子转移是实现高效氧还原反应的主要途径.

具体按哪种反应途径进行与氧分子在催化剂表面 的吸附方式有关. 一般氧分子的吸附方式有 3 种: 端基 式、桥基式和侧基式 ${ }^{[9,10]}$. 对于桥基式和侧基式吸附, 两 个氧原子均吸附在催化剂表面, 当活性位与氧原子间作
用力较强时, $\mathrm{O}-\mathrm{O}$ 键就会发生断裂, 发生四电子转移 反应; 在端基式吸附下，仅有一个氧原子与活性位作用， $\mathrm{O}$ - $\mathrm{O}$ 键不易断裂, 通常发生二电子转移. 因此, 在设 计制备氧还原催化剂时，应考虑氧分子吸附方式的影 响, 力求使反应朝四电子转移方向进行.

\section{2 非水电解液体系}

非水电解液主要是指有机电解液和固态电解液体 系, 目前这两种电解液主要应用于锂空电池当中. 有机 电解液不易挥发, 且不会受析氢等反应的影响; 固体电 解质可以增加电池的热稳定性，防止金属锂的腐蚀 ${ }^{[3]}$. 然而不论是哪种电解液，其工作原理都基于以下反应:

$$
\begin{aligned}
& \text { 负极: } \mathrm{Li} \rightarrow \mathrm{Li}^{+}+\mathrm{e}^{-} \\
& \text {正极: } 2 \mathrm{Li}^{+}+\mathrm{O}_{2}+2 \mathrm{e}^{-} \rightarrow \mathrm{Li}_{2} \mathrm{O}_{2} \\
& \quad 4 \mathrm{Li}^{+}+\mathrm{O}_{2}+4 \mathrm{e}^{-} \rightarrow 2 \mathrm{Li}_{2} \mathrm{O} \\
& \text { 总反应: } 2 \mathrm{Li}+\mathrm{O}_{2} \rightarrow \mathrm{Li}_{2} \mathrm{O}_{2} \quad E^{\mathrm{o}}=2.96 \mathrm{~V} \text { vs. } \mathrm{Li}^{-} \mathrm{Li}^{+} \\
& \quad 4 \mathrm{Li}+\mathrm{O}_{2} \rightarrow 2 \mathrm{Li}_{2} \mathrm{O} E^{\mathrm{o}}=2.91 \mathrm{~V} \text { vs. Li } / \mathrm{Li}^{+}
\end{aligned}
$$

放电时，金属锂失去电子生成锂离子，氧分子得到 电子并结合锂离子生成过氧化锂 $\left(\mathrm{Li}_{2} \mathrm{O}_{2}\right)$ 或氧化锂 $\left(\mathrm{Li}_{2} \mathrm{O}\right)$, 随着电池放电，这些氧化物沉积于空气电极上，从而降 低电池性能. 目前已有研究表明，当有催化剂存在时， 在足够高的充电电压下, $\mathrm{Li}_{2} \mathrm{O}_{2}$ 可以发生析氧反应，从而 实现锂空电池的再充电 ${ }^{[11]}$, 因此在氧还原反应中就需 要更多 $\mathrm{Li}_{2} \mathrm{O}_{2}$ 的产生.

$\mathrm{Li}_{2} \mathrm{O}_{2}$ 和 $\mathrm{Li}_{2} \mathrm{O}$ 的产生与催化剂表面的氧键能大小有 关. Yang 等 ${ }^{[12]}$ 研究了非水体系中的氧还原机理, 认为氧 分子首先接受一个电子形成超氧化物自由基，该自由基 与锂离子结合生成 $\mathrm{LiO}_{2}$, 吸附在催化剂表面. 当催化剂 表面的吸附氧键能较弱时, $\mathrm{LiO}_{2}$ 能够快速得到电子还原 为 $\mathrm{Li}_{2} \mathrm{O}_{2}$, 而当吸附氧键能较强时, 电子转移受到阻碍, $\mathrm{LiO}_{2}$ 会更多地还原为 $\mathrm{Li}_{2} \mathrm{O}$, 如图 $1^{[12]}$ 所示. 因此催化剂 的选择对反应历程起着非常重要的作用.

可以看出, 不同体系氧还原反应机理对催化剂的要 求是不同的. 深入了解氧还原反应机理和不同催化体系 的性质, 对高活性空气电极发展具有重要的指导意义.

\section{Common pathway}

Relative stronger M-O bond dominant pathway

- - Relative weaker M-O bond dominant pathway
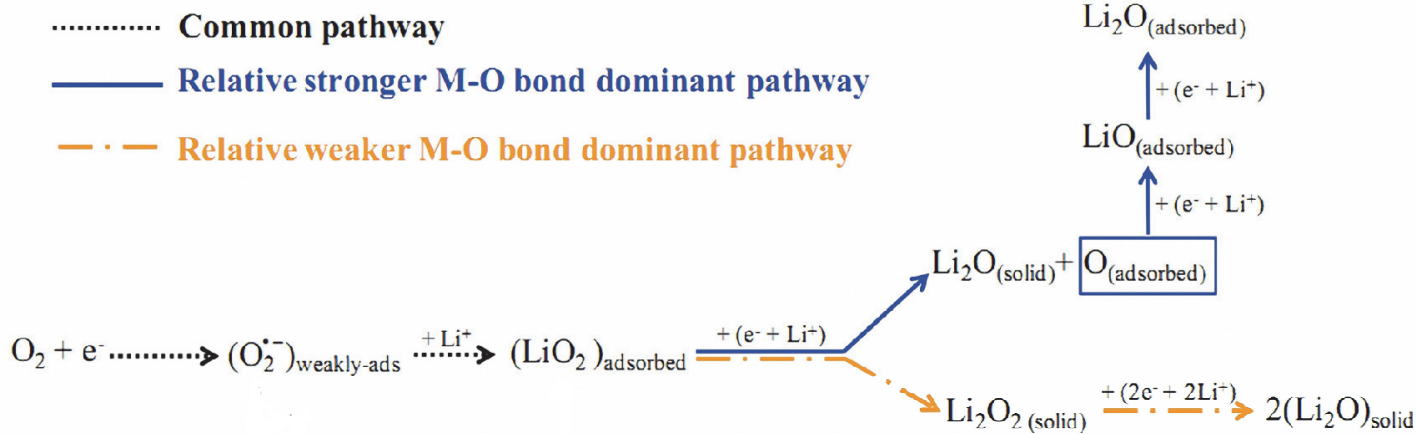

图 1 非水体系氧还原催化机理 ${ }^{[12]}$

Figure 1 ORR mechanism for $\mathrm{Li}^{+}$-containing nonaqueous electrolytes ${ }^{[12]}$ 


\section{3 氧还原催化剂}

近年来, 氧还原催化剂已取得较大发展, 主要包括 贵金属及其合金、金属氧化物、功能化碳材料等.

\section{1 贵金属及其合金}

贵金属尤其是 $\mathrm{Pt}$ 基材料由于在催化氧还原反应中 的高活性和稳定性而被广泛研究, 被认为是水系氧还原 反应中催化性能最好的催化剂, 常被用作评价其它催化 剂活性的标准. 然而 Pt 的成本高且资源短缺, 这就要求 在降低 Pt 用量的同时又要保证高活性和稳定性, 因此 国内外在 Pt 合金方面进行了大量研究. Stamenkovic 等 ${ }^{[13]}$ 对 $\mathrm{Pt}_{3} \mathrm{M}$ ( $\mathrm{M}$ 为 $\mathrm{Fe} 、 \mathrm{Co} 、 \mathrm{Ni} 、 \mathrm{Ti} 、 \mathrm{~V}$ 等)合金的表面 电子结构和催化活性间的关系进行了研究, 发现通过中 间活性物种的吸附能和表面覆盖度间的关系可判断其 在水体系中的催化活性. Nørskov 等 ${ }^{[14]}$ 通过理论计算与 实验进一步证明氧还原催化活性高低可以用氧吸附能 来判断. 一个氧吸附能力比 $\operatorname{Pt}(111)$ 弱 $0 \sim 0.4 \mathrm{eV}$ 的表面 应当具有高于 $\mathrm{Pt}$ 的催化活性, 其最佳值大约在 0.2 $\mathrm{eV}$ (图 2). 同时氧吸附能还与合金形成热存在一定的关 系. 根据以上结论得出 $\mathrm{Pt}_{3} \mathrm{Y}$ 催化活性应当较高, 目前已 有实验结果证明了 Pt-Y 合金的高活性 ${ }^{[15]}$.

Yang 等 ${ }^{[16]}$ 根据在有机体系中氧还原反应机理, 设 计了 $\mathrm{PtAu} / \mathrm{C}$ 双功能催化剂. 在有机体系中, $\mathrm{Au}$ 有利于 催化氧还原反应, Pt 则对氧析出有很好的催化效果, 因 此这样一个双功能催化剂能较好地应用于有机体系的 锂空电池中. 与 $\mathrm{Au} / \mathrm{C}$ 和 $\mathrm{Pt} / \mathrm{C}$ 催化剂相比, $\mathrm{PtAu} / \mathrm{C}$ 提高 了放电比容量, 达 $1500 \mathrm{mAh} / \mathrm{g}$, 平均充电电压仅为 3.6 $\mathrm{V}$, 有效减小了充放电过电位.

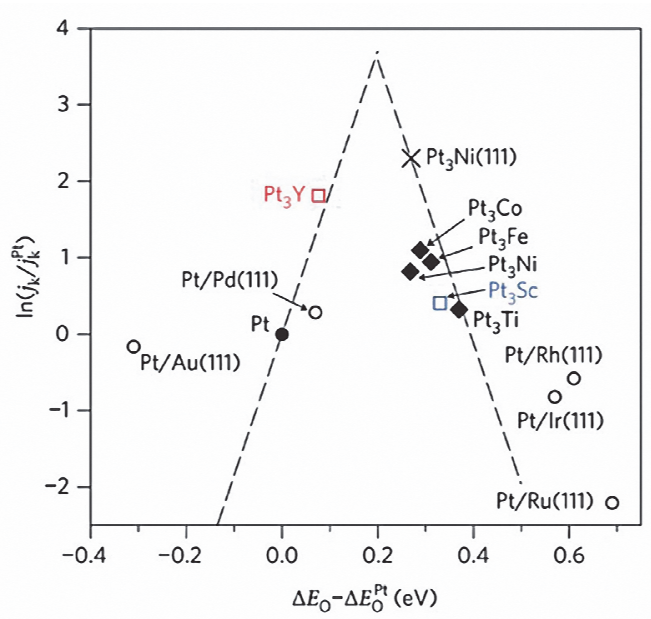

图 2 氧还原动力学电流密度与氧吸附能 $\left(\Delta E_{0}\right)$ 之间的关系 ${ }^{[14]}$

Figure 2 Volcano plots of the oxygen reduction kinetic current density $\left(j_{\mathrm{k}}\right)$ as a function of the calculated oxygen adsorption energy $\left(\Delta E_{\mathrm{o}}\right)^{[14]}$

与 $\mathrm{Pt} 、 \mathrm{Pd}$ 相比, $\mathrm{Ag}$ 资源较为丰富, 价格仅是 $\mathrm{Pt}$ 的 $1 \%$, 在碱性溶液中具有较好的催化稳定性. Shen 等 ${ }^{[17]}$ 通 过间歇微波合成方法制备了 $\mathrm{Ag}-\mathrm{W}_{2} \mathrm{C} / \mathrm{C}$ 催化剂. 与 $\mathrm{Ag} / \mathrm{C} 、 \mathrm{~W}_{2} \mathrm{C} / \mathrm{C}$ 催化剂相比, 该催化剂由于 $\mathrm{Ag}$ 和 $\mathrm{W}_{2} \mathrm{C}$ 间
的协同作用而表现出与 $\mathrm{Pt} / \mathrm{C}$ 相近的半波电位和极限扩 散电流，同时具有很好的抗醇类氧化物的性能，很适合 应用于碱性燃料电池中。一般来说, 纳米尺度较小的颗 粒具有较高的催化活性, 然而 $\mathrm{Ag}$ 催化剂在制备过程中 容易发生团聚，很难得到较小颗粒. Lim 等 ${ }^{[18]}$ 在低温下 制备了高分散和高担载量 $(60 \mathrm{wt} \%)$ 的还原态氧化石墨烯 担载 $\operatorname{Ag}(\mathrm{Ag} / \mathrm{RGO})$. 其中 $\mathrm{Ag}$ 颗粒小于 $10 \mathrm{~nm}$, 且呈现出 高分散均匀性, 但其催化活性仍低于 $\mathrm{Pt} / \mathrm{C}$, 因此 $\mathrm{Ag}$ 催 化剂的催化活性还有待进一步提高.

为降低贵金属成本，往往还可以加入一些过渡金属 氧化物 ${ }^{[19 ~ 22]}$. 过渡金属氧化物一方面可以修饰金属的 电子结构，改变氧分子在其表面的吸附性质；另一方面 可以提高催化剂的电化学稳定性. Woo 等 ${ }^{[23]}$ 通过热分解 方法制备了 $\mathrm{Pd}-\mathrm{Mn}_{3} \mathrm{O}_{4}$ 催化剂. $\mathrm{Mn}_{3} \mathrm{O}_{4}$ 可以将吸附氧传递 给活性物种 $\mathrm{Pd}$, 从而提高催化活性. 通过稳定性测试发 现, $\mathrm{Pd}-\mathrm{Mn}_{3} \mathrm{O}_{4}$ 的面积比活性和质量比活性都比 $\mathrm{Pd} / \mathrm{C}$ 提 高了 $140 \%$ 以上. Deng 等 ${ }^{[24]}$ 考察了 $\mathrm{Co}_{3} \mathrm{O}_{4}$ 对 $\mathrm{Ag} / \mathrm{C}$ 催化性 能的影响, 发现 $\mathrm{Co}_{3} \mathrm{O}_{4}$ 的加入减少了纳米 $\mathrm{Ag}$ 颗粒的团 聚, 增大了催化剂活性面积, 提高了 $\mathrm{Ag} / \mathrm{C}$ 的催化活性. Thapa 等 ${ }^{[25]}$ 通过水热合成方法制备了介孔 $\mathrm{MnO}_{2}$ 担载的 $\mathrm{Au}-\mathrm{Pd}$ 催化剂, 并应用于锂空电池中. 电池放电容量可 达 $775 \mathrm{mAh} / \mathrm{g}$, 在连续 12 次充放电后容量保有率仍可达 $92.1 \%$ ，通过 XRD 和拉曼光谱分析发现放电过程的产物 为 $\mathrm{Li}_{2} \mathrm{O}_{2}$.

\section{2 过渡金属氧化物}

过渡金属氧化物因成本低、环境友好被人们广泛关 注. 过渡金属氧化物种类繁多，大体可分为单一金属氧 化物和复合金属氧化物，其中复合金属氧化物又因其结 构不同可分为尖晶石型、钻钛矿型和烧绿石型.

\section{2 .1 单一金属氧化物}

在单一金属氧化物中, 锰氧化物因其种类较多而被 广泛研究，例如 $\mathrm{MnO} 、 \mathrm{Mn}_{2} \mathrm{O}_{3} 、 \mathrm{MnO}_{2} 、 \mathrm{Mn}_{3} \mathrm{O}_{4}$ 等. 目前 已报道多种锰氧化物可作为氧还原催化剂.

$\mathrm{Mn}_{3} \mathrm{O}_{4}$ 作为其中一种锰氧化物, 具有较高的催化氧 还原性能. 例如离子液体修饰的石墨烯担载的 $\mathrm{Mn}_{3} \mathrm{O}_{4}\left(\mathrm{rGO}-\mathrm{IL} / \mathrm{Mn}_{3} \mathrm{O}_{4}\right)$ 催化剂在锌空电池中显示出较好 的催化活性, 其最大功率密度可达 $120 \mathrm{~mW} / \mathrm{cm}^{2}$, 远高 于商业空气电极. 同时研究发现, 当 $\mathrm{Mn}_{3} \mathrm{O}_{4}$ 担载量较低 时, 氧还原反应机理类似 $\mathrm{Pt} / \mathrm{C}$, 是一个直接四电子转移 过程; 而当担载量较高时, 经历的是两步二电子转 移 ${ }^{[26]}$.

与 $\mathrm{Mn}_{3} \mathrm{O}_{4}$ 相比, $\gamma-\mathrm{MnOOH}$ 具有更高的催化活 性 ${ }^{[27,28]} . \mathrm{MnOOH}$ 不仅能够催化 $\mathrm{O}_{2}^{--}$歧化反应生成 $\mathrm{O}_{2}$ 和 $\mathrm{HO}_{2}^{-}$，同时还能催化 $\mathrm{HO}_{2}^{-}$歧化生成 $\mathrm{O}_{2}$ 和 $\mathrm{OH}^{-[29]}$. Sun 等 ${ }^{[29]}$ 通过考察不同担载量时 $\mathrm{MnOOH} / \mathrm{C}$ 催化剂的催化 活性发现, 随着 $\mathrm{MnOOH}$ 含量的增加, 催化活性不断增 强, 当含量达 $72 \mathrm{wt} \%$ 时, 催化活性最高. 而当担载量继 续增加时, 过多的氧化物使得催化剂导电性降低, 减少 
了电子转移, 从而导致催化活性下降. Zhang 等 ${ }^{[30]}$ 通过 水热合成方法制备了 $\gamma-\mathrm{MnOOH}$ 纳米线, 并应用于锂空 电池中. 与商业碳 $\mathrm{KB}$ 相比, 该催化剂具有相对较低的 充电电压 $(3.8 \mathrm{~V})$, 其放电容量达 $2693 \mathrm{mAh} / \mathrm{g}$, 在连续 10 次循环充放电之后, 电池容量保有率仍达 $72.3 \%$, 表现 出较好的稳定性. 通过对比反应前后氧电极 SEM 图发 现, $\mathrm{MnOOH}$ 的纳米线结构使氧电极更加松散多孔, 为 放电产物提供了更多的空间, 同时也有助于气体传输和 电解液渗透.

与 $\mathrm{MnOOH}$ 类似, $\mathrm{MnO}_{2}$ 同样有着较好的催化性 能 ${ }^{[31,32]}$. 由于氧还原反应是一结构敏感型反应, 因此催 化剂的晶体结构和形貌都会影响其活性. 研究发现对于 $\mathrm{MnO}_{2}$, 其催化活性顺序为 $\alpha->\gamma->\beta-\mathrm{MnO}_{2}{ }^{[33]}$. 即使对 于同一晶型, 不同的形貌结构也显示出不同的催化活 性. Chen 等 ${ }^{[34]}$ 通过不同的制备方法合成了 $\alpha-\mathrm{MnO}_{2}$ 颗 粒、纳米线和花状纳米小球 (图 $3 \mathrm{a}$ 和 $3 \mathrm{~b}$ ). 通过电化学分 析发现, 纳米线和纳米小球具有相对较高的催化活性 (图 3c). 纳米结构具有更大的比表面积和活性位, 提供 了更多催化剂与电解液的接触机会, 同时其特殊的孔道 结构也有利于 $\mathrm{O}_{2}$ 的扩散、吸附和传递. 其次, 催化剂导 电性也是影响催化性能的一个因素. 如图 $3 \mathrm{~d}$ 所示, $\mathrm{Ni}$ 的 加入使得 $\mathrm{MnO}_{2}-\mathrm{NWs} @ \mathrm{Ni}-\mathrm{NPs} / \mathrm{C}$ 显示出更高的催化活 性, 通过导电性测试发现 $\mathrm{MnO}_{2}-\mathrm{NWs} @ \mathrm{Ni}-\mathrm{NPs} / \mathrm{C}$ 的电导 率为 $82.5 \mathrm{~S} / \mathrm{m}, \mathrm{MnO}_{2}-\mathrm{NWs}$ 为 $0.086 \mathrm{~S} / \mathrm{m}$. 导电性越高, 越有利于电子传递, 从而加快反应动力学进程 ${ }^{[34]}$. 最 近, 该课题组通过在氢气气氛下对 $\mathrm{MnO}_{2}$ 热处理得到一 新的催化材料 $\left(\mathrm{H}-\mathrm{MnO}_{2}\right)$. 与原有的 $\mathrm{MnO}_{2}$ 相比, 该催化 剂显示出更高的催化活性、低起始电位和高稳定性. 通
过导电性测试和理论计算, 他们认为此结果主要是因为 催化剂微观结构及电子性质的变化. 催化剂表面氢原子 容易通过氢键的形成而更好地吸附氧分子，从而促进反 应的进行 ${ }^{[35]}$. 同时他们还发现氩气或空气气氛下热处 理后的 $\mathrm{MnO}_{2}$ 拥有更多的氧空穴, 更能提高催化活性 ${ }^{[36]}$. 此外, $\mathrm{MnO}_{2}$ 在非水条件下也具有较好的催化氧还原性 能 ${ }^{[37 ~ 40]}$. Bruce 课题组 ${ }^{[41]}$ 考察了多种锰氧化物在锂空电 池中的应用, 发现 $\alpha-\mathrm{MnO}_{2}$ 纳米线具有最好的催化性能, 在 $75 \mathrm{~mA} / \mathrm{g}$ 放电时电池容量可达 $3000 \mathrm{mAh} / \mathrm{g}$, 并可稳定 循环 10 次, 氧还原电位大约为 $2.75 \mathrm{~V}$ vs. $\mathrm{Li} / \mathrm{Li}^{+}$.

除锰氧化物外, 钴氧化物也是一类较好的氧还原催 化剂 ${ }^{[2244]}$. Dai 等 ${ }^{[45]}$ 通过两步合成方法制备了双功能性 催化剂 $\mathrm{N}$ 掺杂石墨烯担载的 $\mathrm{Co}_{3} \mathrm{O}_{4}\left(\mathrm{Co}_{3} \mathrm{O}_{4} / \mathrm{N}-\mathrm{rmGO}\right)$, 该 催化剂在碱性溶液中表现出高于 $\mathrm{Co}_{3} \mathrm{O}_{4} / \mathrm{rmGO}$ 的催化活 性和高于 $\mathrm{Pt} / \mathrm{C}$ 的稳定性. 通过 $\mathrm{X}$ 射线吸收近边结构 (XANES) 研究发现, 与 $\mathrm{Co}_{3} \mathrm{O}_{4}$ 和 N-rmGO 相比, $\mathrm{Co}_{3} \mathrm{O}_{4} / \mathrm{N}-\mathrm{rmGO}$ 中有 $\mathrm{Co}-\mathrm{N}-\mathrm{C}$ 和 $\mathrm{Co}-\mathrm{O}-\mathrm{C}$ 键的形成, 同时 $\mathrm{O}$ 区有着更高的电子密度, 而 $\mathrm{Co}$ 区电子密度降低, 使得 $\mathrm{Co}-\mathrm{O}$ 键能更高, 因此新键的形成和 $\mathrm{Co} 、 \mathrm{O} 、 \mathrm{C}$ 化 学键环境的改变增强了 $\mathrm{Co}_{3} \mathrm{O}_{4}$ 和 N-rmGO 的协同作用. 其次, $\mathrm{N}$ 掺杂在一定程度上控制了 $\mathrm{Co}_{3} \mathrm{O}_{4}$ 的成核, 减小 了 $\mathrm{Co}_{3} \mathrm{O}_{4}$ 颗粒大小, 从而增加电化学活性面积. 随后他 们又报道了 $\mathrm{CoO} / \mathrm{NCNT}$ (NCNT 为氮修饰的碳纳米管), 其半波电位仅与 $\mathrm{Pt} / \mathrm{C}$ 相差 $35 \mathrm{mV}$, 若将该催化剂涂覆在 碳纤维纸上发现, 该催化剂的电荷转移电阻小于 $\mathrm{Pt} / \mathrm{C}$, 表现出高于 $\mathrm{Pt} / \mathrm{C}$ 的催化活性, 在 $0.7 \mathrm{~V}$ 时其电流密度是 $\mathrm{Pt} / \mathrm{C}$ 的 2 倍. 在 $80{ }^{\circ} \mathrm{C}$ 高浓度碱性溶液中, 该催化剂仍显 示出比 $\mathrm{Pt} / \mathrm{C}$ 高的催化活性和低过电位 ${ }^{[46]}$. Nazar 等 ${ }^{[47]}$ 将
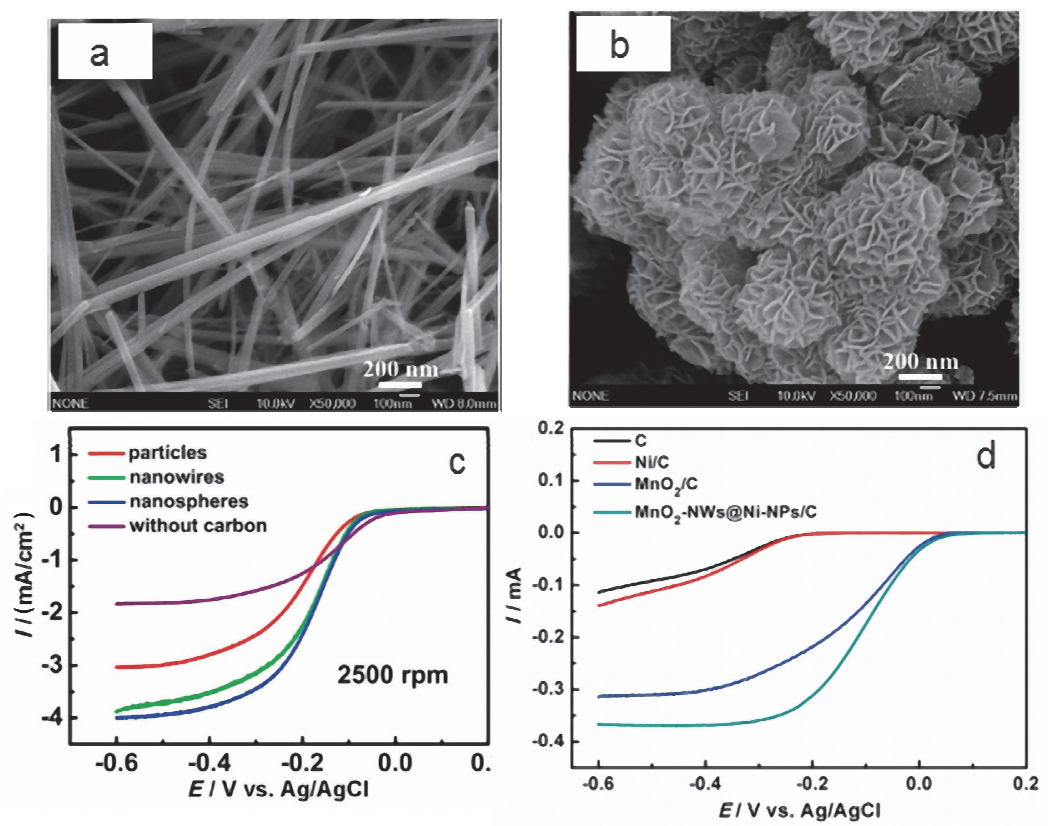

图 $3 \alpha-\mathrm{MnO}_{2}$ 纳米线(a)和花状纳米小球(b)的 SEM 图, (c)不同形貌 $\alpha-\mathrm{MnO}_{2}$ 的极化曲线, (d) $\mathrm{MnO}_{2}-\mathrm{NWs} @ \mathrm{Ni}-\mathrm{NPs} / \mathrm{C}$ 的极化曲线 ${ }^{[34]}$

Figure 3 SEM images of $\alpha-\mathrm{MnO}_{2}$ nanowires (a) and flower-like nanospheres (b), (c) LSV curves of neat $\alpha-\mathrm{MnO}_{2}$ nanowires, bulk particles/carbon, nanowires/carbon, and nanospheres/carbon, (d) LSV curves of C, Ni/C, $\mathrm{MnO}_{2} / \mathrm{C}$, and $\mathrm{MnO}_{2}-\mathrm{NWs} @ \mathrm{Ni}-\mathrm{NPs} / \mathrm{C}^{[34]}$ 
石墨烯担载的 $\mathrm{Co}_{3} \mathrm{O}_{4}$ 应用于有机电解液体系锂空电池 中, 发现 $\mathrm{Co}_{3} \mathrm{O}_{4}$ 的加入有效降低了充电电压, 在连续放 电 7 次后仍保持较高的电池容量.

此外, 其它一些金属氧化物也具有催化氧还原性 能, 例如 $\mathrm{Cu}_{2} \mathrm{O}^{[48]}$. Guo 等 ${ }^{[49]}$ 通过溶剂热方法制备了石墨 烯担载的 $\mathrm{Cu}_{2} \mathrm{O}$, 其中 $\mathrm{Cu}_{2} \mathrm{O}$ 颗粒仅为 $4 \mathrm{~nm}$, 且均匀分布 在石墨烯表面. 通过性能测试发现, 该催化剂催化活性 虽不如 $\mathrm{Pt} / \mathrm{C}$, 但在连续扫描 $16000 \mathrm{~s}$ 后催化活性仍然不 变, 表现出很好的稳定性和抗甲醇能力, 为碱性燃料电 池和金属空气电池的发展提供了新的选择.

\section{2 .2 复合金属氧化物/硫化物}

(1) 尖晶石型氧化物: 钴锰尖晶石型氧化物 $\left(\mathrm{Mn}_{x} \mathrm{Co}_{3-}{ }_{-} \mathrm{O}_{4}\right)$ 由于其较高的催化活性及在碱性溶液中的 高稳定性常被用作空气电极的双功能催化剂 ${ }^{[50}$ - 53]. 传 统制备方法一般需要较高的温度, 虽然晶型较好, 但往 往由于颗粒较大, 比表面积较小而影响催化活性. 近年 来一种室温条件下制备钴锰氧化物的方法取得了较好 的催化活性 ${ }^{[54]}$. 通过还原含有 $\mathrm{Co}^{2+}$ 溶液的 $\mathrm{MnO}_{2}$ 得到的 Co-Mn-O 氧化物具有较高比表面积、多晶格缺陷和空 穴, 与传统制备方法相比, 该方法可以快速选择性地合 成立方或四方型氧化物. 同时研究发现, 立方型氧化物 的催化氧还原性能要高于四方型, 而在氧析出反应中则 相反. 通过对两种不同结构氧化物典型晶面的理论计算 和物理化学表征表明催化性能的不同决定于不同结构 表面的氧键能大小, 立方型表面有更多的 $\mathrm{O}_{\mathrm{ads}}$, 也就是 说有更多的催化活性位, 因此立方型催化氧还原能力高 于四方型. 该结论为进一步设计双功能性催化剂提供了 依据.

Dai 课题组 ${ }^{[55]}$ 在前期研究的基础上采用水热合成方 法制备了氮掺杂石墨烯担载的 $\mathrm{MnCo}_{2} \mathrm{O}_{4}$, 并用于催化氧 还原反应中. 经 XANES 分析得知, 氧化物中部分 $\mathrm{Co}^{3+}$ 区域被 $\mathrm{Mn}^{3+}$ 代替, 增加了催化活性位, 从而提高了催 化活性. 随后他们又将该催化剂用于锂空气电池中 ${ }^{[56]}$, 发现与贵金属 Pt 相比, 该催化剂降低了电池过电位, 延 长了电池循环寿命.

除钴锰氧化物外, $\mathrm{CoFe}_{2} \mathrm{O}_{4}{ }^{[57]} 、 \mathrm{NiCo}_{2} \mathrm{O}_{4}{ }^{[58]}$ 等也被用 作氧还原催化剂. Sun 等 ${ }^{[59]}$ 采用热分解方法制备了单层 分散的 $\mathrm{M}_{x} \mathrm{Fe}_{3-} \mathrm{O}_{4}(\mathrm{M}=\mathrm{Fe}, \mathrm{Cu}, \mathrm{Co}, \mathrm{Mn})$ 纳米颗粒，其中 $\mathrm{Mn}_{x} \mathrm{Fe}_{3-}{ }_{x} \mathrm{O}_{4}$ 表现出较高的催化活性. 通过调节 $\mathrm{Mn}$ 含量 发现, $\mathrm{MnFe}_{2} \mathrm{O}_{4}$ 催化活性较高, 与 $\mathrm{Pt} / \mathrm{C}$ 相当, 同时在碱 性溶液中表现出较好的催化稳定性, 在连续扫描 $9 \mathrm{~h}$ 后 催化活性仍保持 $87 \%$. Cui 等 ${ }^{[60]}$ 将 $\mathrm{NiCO}_{2} \mathrm{O}_{4}$ 介孔纳米片 作为阴极催化剂用于锂空电池中, 与常用的 Super P 碳 黑相比, 该催化剂显示出较高的氧还原起始电位(2.9 $\mathrm{V}$ ), 接近理论值 $2.96 \mathrm{~V}$ ( vs. $\mathrm{Li} / \mathrm{Li}^{+}$), 放电容量达 1560 $\mathrm{mAh} / \mathrm{h}$, 远高于商业碳 Super P (1000 mAh/h), 在连续循 环充放电 10 次, 电池容量保有率仍有 $77.8 \%$.

(2) 钙钛矿型氧化物: 钙钛矿型氧化物种类多样,
在室温下具有很高的导电率, 是较为理想的氧还原催化 剂，常被用于固体氧化物燃料电池中 ${ }^{[61 ~ 64]}$, 其结构式为 $\mathrm{ABO}_{3}$, 其中 $\mathrm{A}$ 为 $\mathrm{La} 、 \mathrm{Ca} 、 \mathrm{Sr} 、 \mathrm{Ba}$ 等, $\mathrm{B}$ 为 $\mathrm{Mn} 、 \mathrm{Co}$ 、 $\mathrm{Ni} 、 \mathrm{Fe}$ 等. 目前研究认为钙钛矿型氧化物的催化活性主 要与 $\mathrm{B}$ 原子 $\sigma^{*}$ 轨道 $\left(e_{\mathrm{g}}\right)$ 电子数及过渡金属和氧间的共价 程度有关. 当 $e_{\mathrm{g}}=1$ 时, 催化活性最高, 如果金属 $3 \mathrm{~d}$ 轨 道与氧 $2 \mathrm{p}$ 轨道共价程度进一步增强, 那么催化活性会 进一步提高 ${ }^{[65]}$. 根据此原理, $\mathrm{LaMnO}_{3+\delta}$ 和 $\mathrm{LaNiO}_{3}$ 在碱 性溶液中显示出与 $\mathrm{Pt} / \mathrm{C}$ 相近的催化活性.

一种由 $\mathrm{LaMnO}_{3}$ 和 $\mathrm{LaNiO}_{3}$ 组成的双功能催化剂显 示出较好的催化氧还原和氧析出性能 ${ }^{[66,67]} . \mathrm{LaNiO}_{3}$ 具有 很好的导电性和催化氧析出能力, $\mathrm{LaMnO}_{3}$ 能够较好地 催化氧还原, 这两种氧化物的结合为催化氧还原和氧析 出反应提供了平衡. 最近, Takeguchi 等 ${ }^{[68]}$ 研究发现 Ruddlesden-Popper 型层状 $\mathrm{LaSr}_{3} \mathrm{Fe}_{3} \mathrm{O}_{10}\left(\mathrm{RP}-\mathrm{LaSr}_{3} \mathrm{Fe}_{3} \mathrm{O}_{10}\right)$ 具有很好的催化氧还原和氧析出性能. 与已报道的 $\mathrm{RuO}_{2}-\mathrm{IrO}_{2}{ }^{[69]}$ 和 $\mathrm{LaMnO}_{3} / \mathrm{LaNiO}_{3}{ }^{[70]}$ 相比, RP-LaSr $3 \mathrm{Fe}_{3} \mathrm{O}_{10}$ 实现了可逆的氧还原和氧析出反应，几乎没有过电位产 生(图 4). 同时该催化剂允许 $\mathrm{H}_{2} \mathrm{O}$ 分子的插入, 形成 $\mathrm{LaSr}_{3} \mathrm{Fe}_{3} \mathrm{O}_{10-2 x}(\mathrm{OH})_{2 x} \cdot \mathrm{H}_{2} \mathrm{O}$, 该氧化物具有低电子电导率 和高离子电导率, 很有希望成为燃料电池电解液. Imanishi 等 ${ }^{[71]}$ 将 $\mathrm{La}_{0.6} \mathrm{Ca}_{0.4} \mathrm{Co}_{0.8} \mathrm{Fe}_{0.2} \mathrm{O}_{3} 、 \mathrm{La}_{0.8} \mathrm{Sr}_{0.2} \mathrm{Fe}_{0.8^{-}}$ $\mathrm{Mn}_{0.2} \mathrm{O}_{3}$ 和 $\mathrm{La}_{0.6} \mathrm{Sr}_{0.4} \mathrm{Co}_{0.2} \mathrm{Fe}_{0.8} \mathrm{O}_{3}$ 作为催化剂用于含 $\mathrm{LiCl}$ 的 $\mathrm{LiOH}$ 溶液的混合式锂空电池中. 发现钙钛矿型氧化 物的加入降低了氧还原和氧析出过电位, 且没有出现电 解液的分解.

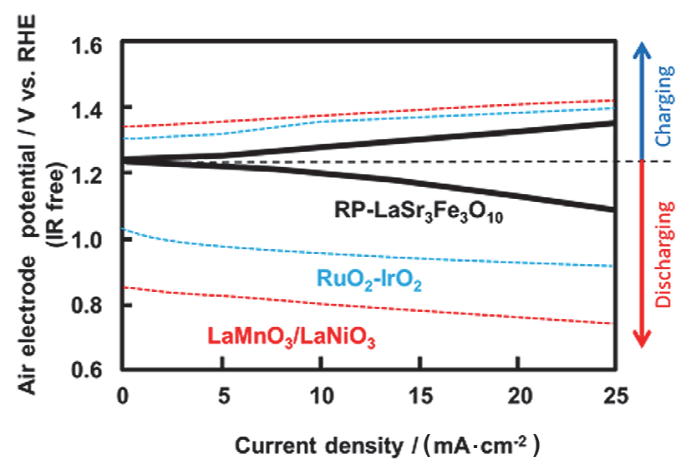

图 4 RP- $\mathrm{LaSr}_{3} \mathrm{Fe}_{3} \mathrm{O}_{10}$ 催化氧还原和氧析出性能 ${ }^{[68]}$

Figure 4 Performance of ORR and OER of RP- $\mathrm{LaSr}_{3} \mathrm{Fe}_{3} \mathrm{O}_{10}{ }^{[68]}$

（3）烧绿石型氧化物: 具有立方结构的烧绿石型氧 化物 $\left(\mathrm{A}_{2} \mathrm{~B}_{2} \mathrm{O}_{6} \mathrm{O}^{\prime}\right)$ 已被用作金属空气电池催化剂 ${ }^{[72]}$, 其中 $\mathrm{A}$ 为 $\mathrm{Pb}$ 或 $\mathrm{Bi}, \mathrm{B}$ 为 $\mathrm{Ru}$ 或 $\mathrm{Ir}^{[73 \sim 75]}$. 八面体结构的 $\mathrm{BO}_{6}$ 相 连形成类似 $\mathrm{B}_{2} \mathrm{O}_{6}$ 结构的笼状结构, 该结构为电子传递 提供了途径, 使此类氧化物具有较高的导电性, 例如 $\mathrm{Pb}_{2} \mathrm{Ru}_{2} \mathrm{O}_{6.5}$, 它在 $300 \mathrm{~K}$ 时电导率达 $4.3 \times 10^{3} \mathrm{~S} / \mathrm{cm}^{[73]}$. 特 殊的氧原子 $\mathrm{O}^{\prime}$ 与 $\mathrm{A}$ 相连形成 $\mathrm{A}-\mathrm{O}^{\prime}-\mathrm{A}, \mathrm{O}^{\prime}$ 位的氧原子可以 全部或部分不存在, 从而导致氧空穴的增加. $\mathrm{Pb}_{2} \mathrm{Ir}_{2} \mathrm{O}_{7-y}$ 作为典型的烧绿石氧化物已被证实是一种较好的催化 氧电化学反应的催化剂. 它在碱性溶液中具有很好地催 
化氧还原性能, 且稳定性较高; 在酸性条件下能很好地 促进氧析出反应的进行 ${ }^{[76]}$. Nazar 等 ${ }^{[77]}$ 采用软模板剂方 法制备了介孔铅钉氧化物, 该催化剂呈现多孔结构, 比 表面积和孔容分别为 $155 \mathrm{~m}^{2} / \mathrm{g}$ 和 $0.18 \mathrm{~cm}^{3} / \mathrm{g}$, 提高了电 化学活性面积, 同时其电导率较高 $(21.7 \mathrm{~S} / \mathrm{cm})$, 有利于 电子传递. 通过研究在有机电解液体系锂空电池中的催 化性能发现, $70 \mathrm{~mA} / \mathrm{g}$ 放电时, 电池放电容量可达 10300 $\mathrm{mAh} / \mathrm{g}$, 有效降低了充电电位 $(3.8 \mathrm{~V})$, 远低于商业碳(4.3 $\mathrm{V})$, 在连续循环充放电 20 次后电池容量仍保持不变.

（4）复合金属硫化物: 近年来已有研究表明具有尖 晶石型结构的硫化物同样能够催化氧还原反应 ${ }^{[78,79]}$. Wang 等 ${ }^{[80]}$ 以 $\mathrm{Na}_{2} \mathrm{~S}$ 为硫源和还原剂一步水热合成法制 备了还原态氧化石墨烯担载的 $\mathrm{NiCo}_{2} \mathrm{~S}_{4}\left(\mathrm{NiCo}_{2} \mathrm{~S}_{4}-\mathrm{rGO}\right)$, 如图 5 所示 $\mathrm{NiCo}_{2} \mathrm{~S}_{4}$ 颗粒没有明显的团聚现象, 通过性 能测试发现其半波电位为 $0.733 \mathrm{~V} \cdot(\mathrm{vs}$. RHE), 远高于 $\mathrm{NiCo}_{2} \mathrm{O}_{4}-\mathrm{rGO}(0.652 \mathrm{~V})$ 和 $\mathrm{rGO}(0.617 \mathrm{~V})$, 仅与 $\mathrm{Pt} / \mathrm{C}$ 相差 $62 \mathrm{mV}$. 他们认为 $\mathrm{NiCo}_{2} \mathrm{~S}_{4}$ 和 $\mathrm{rGO}$ 的协同作用有助于催 化活性的提高, $\mathrm{NiCo}_{2} \mathrm{~S}_{4}$ 结构中更多的活性位 $\mathrm{Co}^{3+}$ 和相 对较高的导电性使得其催化活性高于 $\mathrm{NiCo}_{2} \mathrm{O}_{4}-\mathrm{rGO}$.
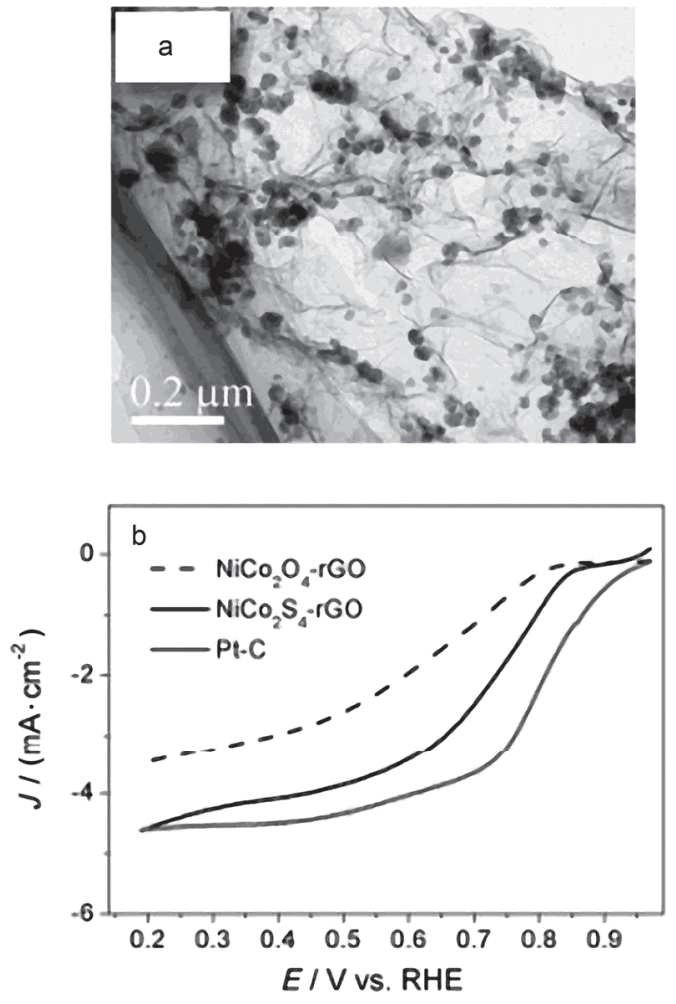

图 5 (a) $\mathrm{NiCo}_{2} \mathrm{~S}_{4}-\mathrm{rGO}$ 的 TEM 图和(b) $\mathrm{NiCo}_{2} \mathrm{~S}_{4}-\mathrm{rGO}$ 催化氧还原性能 曲线 ${ }^{[80]}$

Figure 5 (a) TEM images of $\mathrm{NiCo}_{2} \mathrm{~S}_{4}-\mathrm{rGO}$ and (b) Polarization curve of $\mathrm{NiCO}_{2} \mathrm{~S}_{4}-\mathrm{rGO}^{[80]}$

\section{3 功能化碳材料}

近年来, 功能化碳材料作为最有希望代替 $\mathrm{Pt}$ 的非 贵金属催化剂一直被广泛研究, 主要有金属有机大环化
合物和杂原子掺杂的碳材料.

金属酞菁、金属卟啉等大环化合物由于其较高的共 轭结构和化学稳定性, 对氧分子具有良好的电催化还原 性能 ${ }^{[81]}$. 目前金属有机络合物从合成方法到催化机理 已被广泛研究 ${ }^{[82 ~ 87]}$, 然而其催化本质仍不清楚. 目前普 遍认为催化活性中心涉及到 $\mathrm{C} 、 \mathrm{~N}$ 和过渡金属如 $\mathrm{Fe}$ 、 $\mathrm{Co}^{[88]}$, 但具体到哪一个位置起主导作用, 意见仍不统 一，主要集中在金属上.

Chen 等 ${ }^{[89}$ 通过研究碳载铁酞菁 $(\mathrm{FePc} / \mathrm{C})$ 和钴酞菁 $(\mathrm{CoPc} / \mathrm{C})$ 在碱性电解液中的催化氧还原性能发现, 对于 $\mathrm{FePc} / \mathrm{C}$, 氧还原经历的是四电子转移过程, 而在 $\mathrm{CoPc} / \mathrm{C}$ 上则发生的是二电子转移反应, 且 $\mathrm{FePc} / \mathrm{C}$ 的催化活性 高于 $\mathrm{CoPc} / \mathrm{C}$, 但稳定性较差. 通过理论计算, 他们得出 3 点结论: (1)催化剂表面 $\mathrm{H}_{2} \mathrm{O}_{2}$ 的吸附方式决定了反应途 径(图 6): 对于 $\mathrm{FePc} / \mathrm{C}, \mathrm{H}_{2} \mathrm{O}_{2}$ 以 $\mathrm{HOOH}$ 方式吸附在催化 剂表面, $\mathrm{O}-\mathrm{O}$ 键易断裂, 发生四电子转移; 对于 $\mathrm{CoPc} / \mathrm{C}, \mathrm{H}_{2} \mathrm{O}_{2}$ 以 $\mathrm{H}_{2} \mathrm{OO}$ 方式吸附, 不存在 $\mathrm{O}-\mathrm{O}$ 键断裂 现象, 因此发生二电子转移; (2)催化剂表面的氧吸附影 响反应起始电位: $\mathrm{O}_{2}$ 吸附能越低, 催化活性越高; (3) $\mathrm{OH}$ 的吸附影响催化稳定性: 当催化剂表面吸附更多的 $\mathrm{OH}$ 时, 阻碍了活性位对 $\mathrm{O}_{2}$ 的进一步吸附, 从而导致活性降 低. 由于在 $\mathrm{FePc}$ 表面得到一较大的 $\mathrm{OH}$ 吸附能, 因此 $\mathrm{FePc} / \mathrm{C}$ 的稳定性低于 $\mathrm{CoPc} / \mathrm{C}$. 这说明金属原子在催化 活性、稳定性和反应机理上起着非常重要的作用. 另一 方面，由于一些不含金属原子的化合物也具有催化活 性，因此一些观点认为金属原子对于活性中心并不是必 要的, 而仅仅参与了活性中心的创建 ${ }^{[90]}$. 因此, 对于真 正的催化活性位和反应机理还需要更深入的研究.
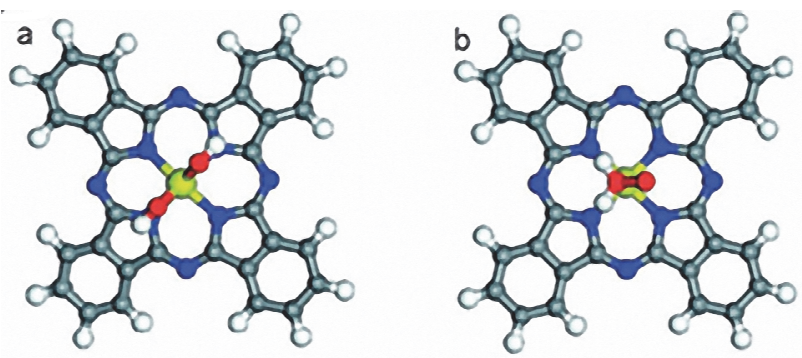

图 $6 \mathrm{H}_{2} \mathrm{O}_{2}$ 在 $\mathrm{FePc}$ (a) 和 $\mathrm{CoPc}$ (b) 上的吸附. $\mathrm{FePc}$ 上吸附的 $\mathrm{HOOH}$, $\mathrm{CoPc}$ 上吸附的 $\mathrm{H}_{2} \mathrm{OO}^{[89]}$

Figure 6 Structural configurations for $\mathrm{HOOH}$ adsorbed on $\mathrm{FePc}$ (a) and $\mathrm{H}_{2} \mathrm{OO}$ adsorbed on $\mathrm{CoPc}(\mathrm{b})^{[89]}$

由于金属有机大环化合物在强酸或强碱中稳定性 较差, 因此高温热处理通常能大大提高其稳定性. 这种 情况下, 寻找合适的热处理条件就成为了研究重点. Dahn 等 ${ }^{[91 ~ 93]}$ 考察了 M-C-N (M 为过渡金属, 如 Mn、Fe、 $\mathrm{Co} 、 \mathrm{Ni}$ ) 材料在酸性和碱性溶液中催化氧还原性能. 发现 在 $700 \sim 900{ }^{\circ} \mathrm{C}$ 催化活性和稳定性最佳, 同时在酸性和 碱性溶液中，不同金属原子使得催化剂活性也有所不 同. 这些结果表明通过调节热处理温度、金属原子等影 响因素可以提高其催化性能. 
除价格较为昂贵且结构复杂的大环化合物外, 杂环 共轭聚合物和胺类化合物等也被应用于催化剂制备中. 由硫酸亚铁、硝酸钴和乙二胺(EDA) 热分解制备的 FeCo-EDA 催化剂在碱性溶液中显示了很高的催化活性 和稳定性 ${ }^{[94]}$. 与 $\mathrm{Pt} / \mathrm{C}$ 催化剂相比, FeCo-EDA 催化剂的 质量比活性是它的近 3 倍, 在稳定性测试后其质量比活 性仍是 $\mathrm{Pt} / \mathrm{C}$ 的 2.5 倍, 在锌空电池测试中发现, $\mathrm{FeCo}-\mathrm{EDA}$ 为催化剂时可得到高于 $\mathrm{Pt} / \mathrm{C}$ 的电流密度和功 率密度.

对于热处理后含 $\mathrm{N}$ 催化剂的活性位, 人们的观点也

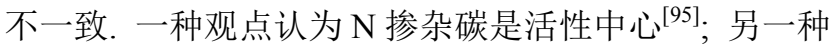
观点认为活性中心是 $M-N_{x}(M \text { 为金属 })^{[96]}$. 最近, Choi 等 ${ }^{[97]}$ 结合实验表征和理论计算考察了 $\mathrm{N}$ 掺杂石墨烯在 水体系中的氧还原反应机理, 认为 $\mathrm{O}_{2}$ 首先在外 Helmholtz 层得到电子生成过氧自由基, 然后再吸附在 催化剂表面进行反应. 同时认为金属物种本身不是活性 中心, 它们存在于石墨烯层之间, 可以将电子转移给石 墨烯层, 提高费米能级, 从而提高催化活性, 因此在设 计制备催化剂时可以适当减小石墨烯尺寸或加入一些 金属物种以提高催化活性.

不论催化剂哪一部分是活性中心位, $\mathrm{N}$ 元素似乎是 催化中心必不可少的一部分, $\mathrm{N}$ 元素含量的增加能够提 高催化活性. 目前通常采用调整含氮前驱体、热分解过 程中通入 $\mathrm{NH}_{3} 、 \mathrm{CH}_{3} \mathrm{CN}$ 或采用新的浸渍方法来提高 $\mathrm{N}$ 含量. 2009 年, Dodelet 等报道了 $\mathrm{Fe}$ 基催化剂, 其催化性 能与 $\mathrm{Pt}$ 相当 ${ }^{[96]}$. 他们采用高微孔结构的 Black Pearl 2000 为载体, 通过将碳载体、1,10-邻菲罗啉和醋酸亚铁 球磨, 并先后在氩气和氨气气氛下热处理而得到了催化 活性较高的 $\mathrm{Fe}$ 基催化剂, 研究发现氨气气氛处理后催 化活性有了很大提高, 通过进一步分析得出拥有较多 $\mathrm{Fe}-\mathrm{N}_{4} / \mathrm{C}(\mathrm{N}$ 为吡啶型和吡咯型)结构的催化剂具有较高 的催化活性 ${ }^{[98]}$.

除 $\mathrm{N}$ 原子能够提高催化活性外, $\mathrm{S} 、 \mathrm{P} 、 \mathrm{~B}$ 等原子的 掺杂也有报道 ${ }^{[99,100]}$. $\mathrm{Hu}$ 等 ${ }^{[101]}$ 考察了 $\mathrm{B}$ 掺杂碳纳米管在 碱溶液中的催化活性, 发现随着 $\mathrm{B}$ 含量的增加, 催化活 性逐渐提高. 理论计算结果表明, 掺杂的 $\mathrm{B}$ 原子起到了 电子传递的作用. 与 $\mathrm{B}$ 形成共轭体系的 $\mathrm{C}$ 原子通过 $\mathrm{B}$ 原 子将 $\pi^{*}$ 电子传递给氧分子, 增强了氧分子的化学吸附, 使 $\mathrm{O}-\mathrm{O}$ 键更易断裂. 他们指出, 作为氧还原催化剂, 制备杂原子掺杂的碳材料需要注意两个因素: (1)不论掺 杂富电子还是缺电子原子, 都需要打破碳材料的电中性 以创造有利于氧分子吸附的区域; (2)充分有效地利用 $\mathrm{C}$ 原子中的 $\pi$ 电子. 此结论为以后设计杂原子掺杂的碳材 料提供了理论依据.

在非水体系中功能化碳材料的应用也有报道. Liu 等 ${ }^{[102]}$ 研究了 Black Pearls 2000 碳黑、醋酸亚铁和 1,10邻菲罗啉热处理制备的 $\mathrm{Fe} / \mathrm{N} / \mathrm{C}$ 催化剂在锂空电池中的 应用. 发现与 $\alpha-\mathrm{MnO}_{2} / \mathrm{C}$ 催化剂相比, 该催化剂降低了
电池过电位，提高了电池效率。通过气相分析发现以 $\mathrm{Fe} / \mathrm{N} / \mathrm{C}$ 为催化剂时未发现 $\mathrm{CO}_{2}$ 的产生, 这说明与促进电 解液分解相比, $\mathrm{Fe} / \mathrm{N} / \mathrm{C}$ 催化剂更能催化过氧化锂的分解 (图 7). 通过扫描透射电镜和 X 射线吸收发现 $\mathrm{Fe}$ 原子级 均匀分布在碳载体表面，有利于减少电子传递和传质的 影响, 因此在充电时表现出较低的过电位. 由此可以看 出, 功能化碳材料对于金属空气电池是一类很有应用潜 力的催化剂.
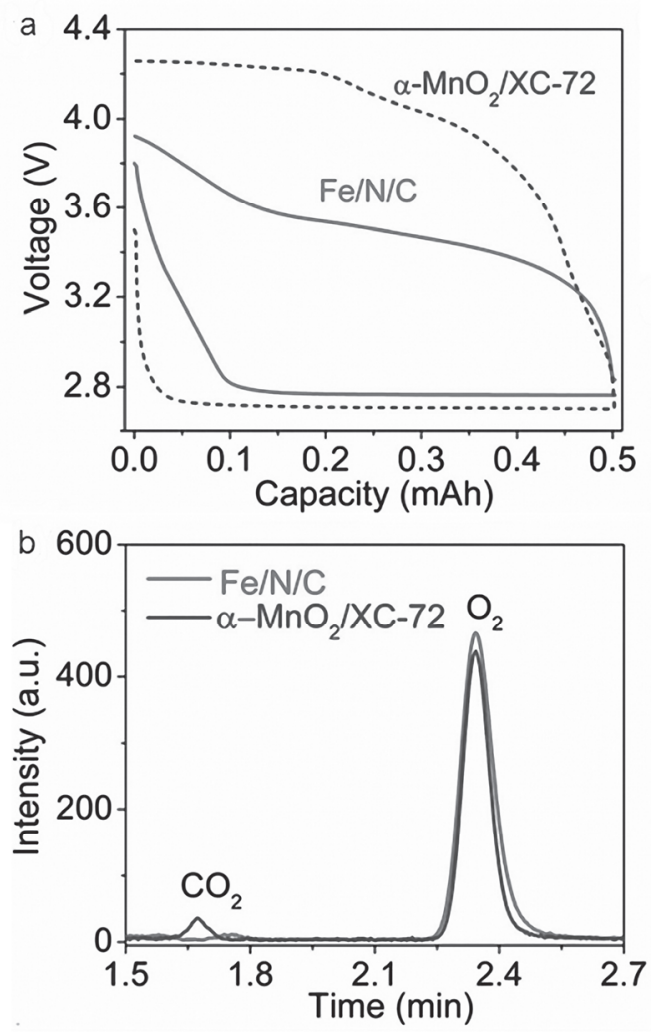

图 $7 \mathrm{Fe} / \mathrm{N} / \mathrm{C}$ 在锂空电池中的应用(a)及气相分析结果(b) ${ }^{[102]}$ Figure 7 Discharge/charge of $\mathrm{Li}^{-} \mathrm{O}_{2}$ cells using $\mathrm{Fe} / \mathrm{N} / \mathrm{C}$ as cathode catalysts (a) and GC signals as a function of retention time (b) ${ }^{[102]}$

\section{4 金属氮化物}

除上述几种催化剂外，金属氮化物也是一类氧还原 催化剂 ${ }^{[103 ~ 105]}$. 此类化合物稳定性高且导电性好, 已被 应用于金属空气电池中. Zhou 等 ${ }^{[106]}$ 考察了活性炭担载 的氮化钛 (TiN/VC) 在有机电解液体系锂空电池中的性 能, 发现与活性炭(VC)相比, TiN/VC 显示出更大的氧还 原电流和更低的氧析出电位, 平均充电电压仅为 $3.5 \mathrm{~V}$, 放电容量可达 $6407 \mathrm{mAh} / \mathrm{g}$ ，这表明金属氮化物在金属 空气电池中是一类有效的催化氧还原和氧析出的双功 能催化剂.

为了更清晰对比各种氧还原催化剂的催化性能, 现 将一些典型催化剂的电化学活性参数列在表 1 和表 2 中. 可以看出, 各种催化剂各有其特点. 在水系电解液中, 
表 1 各种典型催化剂在水系电解液中的性能对比

Table 1 The ORR performance of the main catalysts in aqueous electrolyte

\begin{tabular}{|c|c|c|c|c|c|}
\hline Catalyst & Electrolyte & $\begin{array}{l}\text { Onset potential } \\
\text { (V vs. RHE) }\end{array}$ & $\begin{array}{l}\text { Half wave potential } \\
\qquad \text { (V vs. RHE) }\end{array}$ & $\begin{array}{c}\text { The number of electrons } \\
\text { transferred }\end{array}$ & $\begin{array}{l}\text { Limiting diffusion current } \\
\text { density at } 1600 \mathrm{r} / \mathrm{min} \\
\left(\mathrm{mA} / \mathrm{cm}^{2}\right)\end{array}$ \\
\hline $\mathrm{Pt} / \mathrm{C}^{[45]}$ & $0.1 \mathrm{~mol} / \mathrm{L} \mathrm{KOH}$ & 1.0 & 0.86 & 4.0 & 5.7 \\
\hline $\mathrm{Ag}-\mathrm{W}_{2} \mathrm{C} / \mathrm{C}^{[17]}$ & $1 \mathrm{~mol} / \mathrm{L} \mathrm{KOH}$ & 0.95 & 0.82 & 3.9 & $4.0(1500 \mathrm{r} / \mathrm{min})$ \\
\hline $\mathrm{rGO}-\mathrm{IL} / \mathrm{Mn}_{3} \mathrm{O}_{4}{ }^{[26]}$ & $0.1 \mathrm{~mol} / \mathrm{L} \mathrm{KOH}$ & 0.80 & 0.65 & 3.5 & 3.8 \\
\hline MnO-NWs@Ni-NPs/C ${ }^{[34]}$ & $0.1 \mathrm{~mol} / \mathrm{L} \mathrm{KOH}$ & 0.98 & 0.81 & 3.86 & $0.365^{a}$ \\
\hline $\mathrm{Co}_{3} \mathrm{O}_{4} / \mathrm{N}-\mathrm{rmGO}^{[45]}$ & $0.1 \mathrm{~mol} / \mathrm{L} \mathrm{KOH}$ & 0.93 & 0.83 & 4.0 & 5.0 \\
\hline $\mathrm{Co}_{x} \mathrm{Mn}_{3-x} \mathrm{O}_{4}{ }^{[54]}$ & $0.1 \mathrm{~mol} / \mathrm{L} \mathrm{KOH}$ & 0.91 & - & 3.7 & - \\
\hline $\mathrm{RP}-\mathrm{LaSr}_{3} \mathrm{Fe}_{3} \mathrm{O}_{10}{ }^{[68]}$ & $0.1 \mathrm{~mol} / \mathrm{L} \mathrm{NaOH}$ & 1.23 & 0.6 & - & $5.3(1500 \mathrm{r} / \mathrm{min})$ \\
\hline $\mathrm{NiCo}_{2} \mathrm{~S}_{4}-\mathrm{rGO}^{[80]}$ & $0.1 \mathrm{~mol} / \mathrm{L} \mathrm{KOH}$ & 0.92 & 0.73 & 3.93 & 4.7 \\
\hline FeCo-EDA $^{[94]}$ & $0.1 \mathrm{~mol} / \mathrm{L} \mathrm{KOH}$ & 0.97 & 0.81 & - & 5.7 \\
\hline
\end{tabular}

${ }^{a} 0.365 \mathrm{~mA}$ at a rotation rate of $900 \mathrm{r} / \mathrm{min}$.

表 2 各种典型催化剂在非水水系电解液中的性能对比

Table 2 The ORR performance of the main catalysts in nonaqueous electrolyte

\begin{tabular}{|c|c|c|c|c|}
\hline Catalyst & Average discharge voltage/V & $\begin{array}{l}\text { The first discharge capacity } \\
\qquad\left(\mathrm{mAh} / \mathrm{g}_{\text {carbon }}\right)\end{array}$ & Average charge voltage/ $\mathrm{V}$ & $\begin{array}{c}\text { Capacity retention after } 10 \\
\text { cycles }\end{array}$ \\
\hline $\mathrm{PtAu} / \mathrm{C}^{[16]}$ & 2.7 & $1500\left(100 \mathrm{~mA} / \mathrm{g}_{\text {carbon }}\right)$ & 3.6 & - \\
\hline$\gamma$-MnOOH nanowires ${ }^{[30]}$ & 2.8 & $2693\left(0.05 \mathrm{~mA} / \mathrm{cm}^{2}\right)$ & 3.8 & $72.3 \%$ \\
\hline $\mathrm{NiCo}_{2} \mathrm{O}_{4}$ nanoflakes ${ }^{[60]}$ & 2.6 & $1673\left(0.1 \mathrm{~mA} / \mathrm{cm}^{2}\right)$ & 3.9 & $77.8 \%$ \\
\hline $\mathrm{Pb}_{2}^{2+}\left[\mathrm{Ru}_{1.6} \mathrm{~Pb}_{0.4}{ }^{4+}\right] \mathrm{O}_{6.5}{ }^{[77]}$ & 2.7 & $10300\left(70 \mathrm{~mA} / \mathrm{g}_{\text {carbon }}\right)$ & 3.8 & $\approx 100 \%^{a}$ \\
\hline $\mathrm{Fe} / \mathrm{N} / \mathrm{C}^{[102]}$ & 2.8 & - & 3.6 & $\approx 100 \%$ \\
\hline $\mathrm{n}-\mathrm{TiN} / \mathrm{VC}^{[106]}$ & 2.7 & $6407\left(500 \mathrm{~mA} / \mathrm{g}_{\text {carbon }}\right)$ & 3.7 & - \\
\hline
\end{tabular}

${ }^{a}$ At the end of the $25^{\text {th }}$ cycle.

贵金属系列催化活性相对较高; 在非水系电解液中, 金 属氧化物催化剂具有较高的电池容量, 但充放电过电位 也相对较高. 因此, 在寻找和设计合适催化剂时应考虑 反应体系和不同种类催化剂的特点.

\section{4 结论与展望}

经国内外广泛研究, 金属空气电池氧还原催化剂已 取得长足进步, 但总体上仍有许多问题亟待解决, 比如 氧还原反应机理仍不清楚; 贵金属及其合金的成本及稳 定性方面需要进一步研究; 功能化碳材料中催化活性位 仍不明确; 非 Pt 催化剂的催化活性有待进一步提高等. 针对目前所存在的问题, 未来金属空气电池氧还原催化 剂的研究主要集中在: (1)理论计算与实验相结合, 深入 研究催化剂上氧还原反应机理, 明确各种催化剂活性 位; (2)研究催化剂组成、形貌、比表面积等对催化活性 的影响, 通过改善催化剂制备方法, 优化制备条件, 以 提高催化活性和稳定性; (3)通过氧还原反应机理设计开 发新型高催化性能和稳定性的氧还原催化剂, 为金属空 气电池的商业化发展提供有力保障.

\section{作者简介}

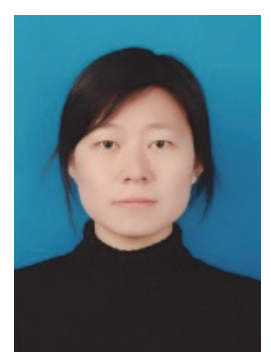

王瀛, 女, 博士, 硕士生导师, 毕业于中国科学院兰州化 学物理研究所, 主要从事金属空气电池阴极材料的制备与性 能研究，现主持校自然基金 1 项.

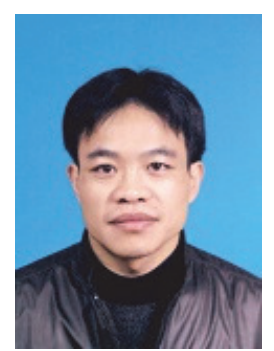

张丽敏, 男, 博士, 副教授、硕士生导师, 2008 年获中国 科学院大连化学物理研究所理学博士学位, 主要从事固体氧 
化物燃料电池电极材料的研究及应用. 主持并完成国家自然 科学基金项目 1 项, 山西省青年科学基金项目 1 项.

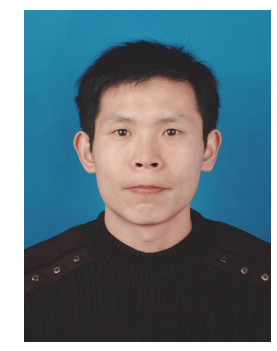

胡天军, 男, 硕士, 毕业于郑州大学, 主要从事绿色催化 方面的研究.

\section{References}

[1] Arora, P.; Zhang, Z. J. Chem. Rev. 2004, 104, 4419.

[2] Bruce, P. G.; Freunberger, S. A.; Hardwick, L. J.; Tarascon, J.-M. Nat. Mater. 2012, 11, 19.

[3] Gu, D.-M.; Zhang, C.-M.; Gu, S.; Zhang, Y.; Wang, Y.; Qiang, L.-S. Acta Chim. Sinica 2012, 70, 2115. (顾大明, 张传明, 顾硕, 张音, 王余，强亮生，化学学报, 2012, 70, 2115.)

[4] Gu, D.-M.; Wang, Y.; Gu, S.; Zhang, C.-M.; Yang, D.-D. Acta Chim. Sinica 2013, 71, 1354. (顾大明, 王余, 顾硕, 张传明, 杨丹丹，化 学学报, 2013, 71, 1354.)

[5] Jiang, J.; Liu, X.; Zhao, S.; He, P.; Zhou, H. Acta Chim. Sinica 2014, 72, 417. (蒋颕, 刘晓飞, 赵世勇, 何平, 周豪慎, 化学学报, 2014, $72,417$.

[6] Cheng, F.; Chen, J. Chem. Soc. Rev. 2012, 41, 2172

[7] Cheng, F.; Chen, J. Acta Chim. Sinica 2013, 71, 473. (程方益, 陈 军, 化学学报, 2013, 71, 473.)

[8] Wang, Z.; Xu, D.; Xu, J.; Zhang, X. Chem. Soc. Rev. 2014, 43, 7746.

[9] Spendelow, J. S.; Wieckowshi, A. Phys. Chem. Chem. Phys. 2007, 9, 2654.

[10] Zinola, C. F.; Arvia, A. J.; Estiu, G. L.; Castro, E. A. J. Phys. Chem. 1994, 98, 7566.

[11] Ogasawara, T.; Debart, A.; Holzapfel, M.; Novak, P.; Bruce, P. G. J. Am. Chem. Soc. 2006, 128, 1390

[12] Lu, Y.-C.; Gasteiger, H. A.; Yang, S.-H. J. Am. Chem. Soc. 2011, $133,19048$.

[13] Stamenkovic, V. R.; Mun, B. S.; Arenz, M.; Mayrhofer, K. J. J.; Lucas, C. A.; Wang, G.; Ross, P. N.; Markovic, N. M. Nat. Mater. 2007, $6,241$.

[14] Greeley, J.; Stephens, I. E. L.; Bondarenko, A. S.; Johansson, T. P.; Hansen, H. A.; Jaramillo, T. F.; Rossmeisl, J.; Chorkendorff, I.; NØrskov, J. K. Nat. Chem. 2009, 1, 552.

[15] Yoo, S. J.; Kim, S. K.; Jeon, T. Y.; Hwang, S. J.; Lee, J. G.; Lee, S. C.; Lee, K. S.; Cho, Y. H.; Sung, Y. E.; Lim, T. H. Chem. Commun. 2011, 47, 11414.

[16] Li, Y. C.; Xu, Z. C.; Gasteiger, H. A.; Chen, S.; Hamad-Schifferli, K.; Yang, S.-H. J. Am. Chem. Soc. 2010, 132, 12170.

[17] Meng, H.; Shen, P. K. Electrochem. Commun. 2006, 8, 588.

[18] Lim, E. J.; Choi, S. M.; Seo, M. H.; Kim, Y.; Lee, S.; Kim, W. B. Electrochem. Commun. 2013, 28, 100.

[19] Lim, D. H.; Lee, W. D.; Choi, D. H.; Lee, H. I. Appl. Catal. B 2010, $94,85$.

[20] Maheswari, S.; Sridhar, P.; Pitchumani, S. Electrochem. Commun. 2013, 26, 97.

[21] Sun, W.; Hsu, A.; Chen, R. J. Power Sources 2011, 196, 4491.

[22] Kim, K. W.; Kim, S. M.; Choi, S.; Kim, J.; Lee, I. S. ACS Nano 2012, 6,5122 .

[23] Choi, C. H.; Park, S. H.; Woo, S. I. Phys. Chem. Chem. Phys. 2012 , 14,6842 .

[24] Wang, Y.; Lu, X.; Liu, Y.; Deng, Y. Electrochem. Commum. 2013, 31,108 .

[25] Thapa, A. K.; Shin, T. H.; Ida, S.; Sumanasekera, G. U.; Sunkara, M. K.; Ishihara, T. J. Power Sources 2012, 220, 211.

[26] Lee, J.-S.; Lee, T.; Song, H.-K.; Cho, J.; Kim, B.-S. Energy Environ. Sci. 2011, 4, 4148

[27] Lanqun, M.; Sotomura, T.; Nakatsu, K.; Koshiba, N.; Dun, Z.;
Ohsaka, T. J. Electrochem. Soc. 2002, 149, A504.

[28] Jin, Q.; Pei, L.; Hu, Y.; Du, J.; Han, X.; Cheng, F.; Chen, J. Acta Chim. Sinica 2014, 72, 920. (靳琪, 裴龙凯, 胡宇翔, 杜婧, 韩晓 鹏, 程方益, 陈军, 化学学报, 2014, 72, 920.)

[29] Sun, W.; Hsu, A.; Chen, R. J. Power Sources 2011, 196, 627.

[30] Zhang, L.; Zhang, X.; Wang, Z.; Xu, J.; Xu, D.; Wang, L. Chem. Commun. 2012, 48, 7598.

[31] Xiao, W.; Wang, D.; Lou, X. W. J. Phys. Chem. C 2010, 114, 1694.

[32] Valim, R. B.; Santos, M. C.; Lanza, M. R. V.; Machado, S. A. S.; Lima, F. H. B.; Calegaro, M. L. Electrochim. Acta 2012, 85, 423.

[33] Cao, Y. L.; Yang, H. X.; Ai, X. P.; Xiao, L. F. J. Electroanal. Chem. 2003, 557, 127 .

[34] Cheng, F.; Su, Y.; Liang, J.; Tao, Z.; Chen, J. Chem. Mater. 2010, 22 , 898.

[35] Zhang, T.; Cheng, F.; Du, J.; Hu, Y.; Chen, J. Adv. Energy Mater. 2014, 1400654.

[36] Cheng, F.; Zhang, T.; Zhang, Y.; Du, J.; Han, X.; Chen, J. Angew. Chem., Int. Ed. 2013, 52, 2474

[37] Li, J.; Wang, N.; Zhao, Y.; Ding, Y.; Guan, L. Electrochem. Commun. 2011, 13, 698.

[38] Cao, Y.; Wei, Z.; He, J.; Zang, J.; Zhang, Q.; Zheng, M.; Dong, Q. Energy Environ. Sci. 2012, 5, 9765.

[39] Qin, Y.; Lu, J.; Du, P.; Chen, Z.; Ren, Y.; Wu, T.; Miller, J. T.; Wen, J.; Miller, D. J.; Zhang, Z.; Amine, K. Energy Environ. Sci. 2013, 6, 519.

[40] Benbow, E. M.; Kelly, S. P.; Zhao, L.; Reutenauer, J. W.; Suib, S. L. J. Phys. Chem. C 2011, 115, 22009.

[41] Debart, A.; Paterson, A. J.; Bao, J.; Bruce, P. G. Angew. Chem., Int. Ed. 2008, 47, 4521.

[42] Guo, S.; Zhang, S.; Wu, L.; Sun, S. Angew. Chem., Int. Ed. 2012, 51, 11770.

[43] Liu, J.; Jiang, L.; Tang, Q.; Zhang, B.; Su, D. S.; Wang, S.; Sun, G. ChemSusChem 2012, 5, 2315.

[44] Sa, Y. J.; Kwon, K.; Cheon, J. Y.; Kleitz, F.; Joo, S. H. J. Mater. Chem. A 2013, 1, 9992.

[45] Liang, Y.; Li, Y.; Wang, H.; Zhou, J.; Wang, J.; Regier, T.; Dai, H. J. Nat. Mater. 2011, 10, 780

[46] Liang, Y.; Wang, H.; Diao, P.; Chang, W.; Hong, G.; Li, Y.; Gong, M.; Xie, L.; Zhou, J.; Wang, J.; Regier, T. Z.; Wei, F.; Dai, H. J. J. Am. Chem. Soc. 2012, 134, 15849.

[47] Black, R.; Lee, J.-H.; Adams, B.; Mims, C. A.; Nazar, L. F. Angew. Chem., Int. Ed. 2012, 51, 1 .

[48] Li, Q.; Xu, P.; Zhang, B.; Tsai, H.; Zheng, S.; Wu, G.; Wang, H.-L. J. Phys. Chem. C 2013, 117, 13872.

[49] Yan, X.-Y.; Tong, X.-L.; Zhang, Y.-F.; Han, X.-D.; Wang, Y.-Y.; Jin, G.-Q.; Qin, Y.; Guo, X.-Y. Chem. Commun. 2012, 48, 1892.

[50] Ríos, E.; Reyes, H.; Ortiz, J.; Gautier, J. L. Electrochim. Acta 2005, $50,2705$.

[51] Koninck, M. D.; Poirier, S. C.; Marsan, B. J. Electrochem. Soc. 2006, 153, A2103.

[52] Nikolova, V.; Iliev, P.; Petrov, K.; Vitanov, T.; Zhecheva, E.; Stoyanova, R.; Valov, I.; Stoychev, D. J. Power Sources 2008, 185, 727.

[53] Du, J.; Pan, Y.; Zhang, T.; Han, X.; Cheng, F.; Chen, J. J. Mater. Chem. 2012, 22, 15812

[54] Cheng, F. Y.; Shen, J.; Peng, B.; Pan, Y. D.; Tao, Z. L.; Chen, J. Nat. Chem. 2010, 3, 79 .

[55] Liang, Y.; Wang, H.; Zhou, J.; Li, Y.; Wang, J.; Regier, T.; Dai, H. J. J. Am. Chem. Soc. 2012, 134, 3517.

[56] Wang, H.; Yang, Y.; Liang, Y.; Zheng, G.; Li, Y.; Cui, Y.; Dai, H. J. Energy Environ. Sci. 2012, 5, 7931.

[57] Bian, W.; Yang, Z.; Strasser, P.; Yang, R. J. Power Sources 2014, $250,196$.

[58] Liu, Z.-Q.; Xu, Q.-Z.; Wang, J.-Y.; Li, N.; Guo, S.-H.; Su, Y.-Z; Wang, H.-J.; Zhang, J.-H.; Chen, S. Int. J. Hydrogen Energy 2013, $38,6657$.

[59] Zhu, H.; Zhang, S.; Huang, Y.-X.; Wu, L.; Sun, S. Nano Lett. 2013, 13,2947

[60] Zhang, L.; Zhang, S.; Zhang, K.; Xu, G.; He, X.; Dong, S.; Liu, Z.; Huang, C.; Gu, L.; Cui, G. Chem. Commun. 2013, 49, 3540.

[61] Yuasa, M.; Imamura, H.; Nishida, M.; Kida, T.; Shimanoe, K. Electrochem. Commun. 2012, 24, 50.

[62] Sunarso, J.; Torriero, A. A. J.; Zhou, W.; Howlett, P. C.; Forsyth, M. J. Phys. Chem. C 2012, 116, 5827

[63] Wang, Y.; Cui, X.; Li, Y.; Chen, L.; Shu, Z.; Chen, H.; Shi, J. Dalton 
Trans. 2013, 42, 9448.

[64] Malavasi, L.; Fisher, C. A. J.; Islam, M. S. Chem. Soc. Rev. 2010, $39,4370$.

[65] Suntivich, J.; Gasteiger, H. A.; Yabuuchi, N.; Nakanishi, H.; Goodenough, J. B.; Yang, S.-H. Nat. Chem. 2011, 3, 546.

[66] Yuasa, M.; Nishida, M.; Kida, T.; Yamazoe, N.; Shimanoe, K. J. Electrochem. Soc. 2011, 158, A605.

[67] Yuasa, M.; Imamura, H.; Nishida, M.; Kida, T.; Shimanoe, K. Electrochem. Commun. 2012, 24, 50.

[68] Takeguchi, T.; Yamanaka, T.; Takahashi, H.; Watanabe, H.; Kuroki, T.; Nakanishi, H.; Orikasa, Y.; Uchimoto, Y.; Takano, H.; Ohguri, N.; Matsuda, M.; Murota, T.; Uosaki, K.; Ueda, W. J. Am. Chem. Soc. 2013, 135, 11125.

[69] Yuasa, M.; Nishida, M.; Kida, T.; Yamazoe, N.; Shimanoe, K. J. Electrochem. Soc. 2011, 158, A605.

[70] Zhang, Y.; Wang, C.; Wan, N.; Mao, Z. Int. J. Hydrogen Energy 2007, 32, 400 .

[71] Ohkuma, H.; Uechi, I.; Imanishi, N.; Hirano, A.; Takeda, Y.; Yamamoto, O. J. Power Sources 2013, 223, 319.

[72] Prakash, J.; Tryk, D.; Yeager, E. J. Power Sources 1990, 29, 413.

[73] Akazawa, T.; Inaguma, Y.; Katsumata, T.; Hiraki, K.; Takahashi, T. J. Cryst. Growth 2004, 271, 445.

[74] Horowitz, H. S.; Longo, J. M.; Lewandowski, J. T. Mater. Res. Bull. 1981, 16, 489

[75] Beyerlein, R. A.; Horowitz, H. S.; Longo, J. M. J. Solid State Chem. 1988, 72, 2.

[76] Goodenough, J. B.; Manoharan, R.; Paranthaman, M. J. Am. Chem. Soc. 1990, 112, 2076.

[77] Oh, S. H.; Black, R.; Pomerantseva, E.; Lee, J.-H.; Nazar, L. F. Nat. Chem. 2012, 4, 1004.

[78] Zhang, Z.; Wang, X.; Cui, G.; Zhang, A.; Zhou, X.; Xu H.; Gu, L.; Nanoscale 2014, 6, 3540 .

[79] Liu, Q.; Jin, J. T.; Zhang, J. Y. ACS Appl. Mater. Interfaces 2013, 5, 5002.

[80] Wu, J.; Dou, S.; Shen, A.; Wang, X.; Ma, Z.; Ouyang, C.; Wang, S. J. Mater. Chem. A 2014, 2, 20990.

[81] Yuan, X.; Xia, X.; Zeng, X.; Zhang, H.; Ma, Z. Prog. Chem. 2010, 22,19 . (原鲜霞, 夏小芸, 曾金鍂, 张慧娟, 马紫峰, 化学进展, 2010, 22, 19.)

[82] Oh, S. H.; Nazar, L. F. Adv. Energy Mater. 2012, 2, 903.

[83] Morozan, A.; Jousselme, B.; Palacin, S. Energy Environ. Sci. 2011, 4, 1238.

[84] Chen, Z. W.; Higgins, D.; Yu, A. P.; Zhang, L.; Zhang, J. J. Energy Environ. Sci. 2011, 4, 3167.

[85] Liu, G.; Li, X.; Lee, J. W.; Popov, B. N. Catal. Sci. Technol. 2011, 1, 207.

[86] Bezerra, C. W. B.; Zhang, L.; Lee, K.; Liu, H. S.; Marques, A. L. B.;
Marques, E. P.; Wang, H. J.; Zhang, J. J. Electrochim. Acta 2008, 53, 4937.

[87] Jaouen, F.; Proietti, E.; Lefèvre, M.; Chenitz, R.; Dodelet, J. P.; Wu, G.; Chung, H. T.; Johnston, C. M.; Zelanay, P. Energy Environ. Sci. 2011, 4, 114.

[88] Lefèvre, M.; Dodelet, J. P.; Bertrand, P. J. Phys. Chem. B 2005, 109, 16718.

[89] Chen, R. R.; Li, H. X.; Chu, D.; Wang, G. F. J. Phys. Chem. C 2009, 113, 20689.

[90] Kobayashi, M.; Niwa, H.; Harada, Y.; Horiba, K.; Oshima, M.; Ofuchi, H.; Terakura, K.; Ikeda, T.; Koshigoe, Y.; Ozaki, J. I.; Miyata, S.; Ueda, S.; Yamashita, Y.; Yoshikawa, H.; Kobayashi, K. J. Power Sources 2011, 196, 8346.

[91] Easton, E. B.; Yang, R.; Bonakdarpour, A.; Dahn, J. R. Electrochem. Solid-State Lett. 2007, 10, B6.

[92] Yang, R. Z.; Stevens, K.; Dahn, J. R. J. Electrochem. Soc. 2008, 155, B79.

[93] Easton, E. B.; Bonakdarpour, A.; yang, R. Z.; Stevens, D. A.; Dahn, J. R. J. Electrochem. Soc. 2008, 155, B547.

[94] Chen, Z.; Choi, J. Y.; Wang, H. J.; Li, H.; Chen, Z. W. J. Power Sources 2011, 196, 3673.

[95] Von Deak, D.; Singh, D.; Biddinger, E. J.; King, J. C.; Bayram, B.; Miller, J. T.; Ozkan, U. S. J. Catal. 2012, 285, 145.

[96] Lefèvre, M.; Proietti, E.; Jaouen, F.; Dodelet, J. P. Science 2009, 324,71 .

[97] Choi, C. H.; Lim, H.-K.; Chung, M. W.; Park, J. C.; Shin, H.; Kim, H.; Woo, S. I. J. Am. Chem. Soc. 2014, 136, 9070.

[98] Kramm, U. I.; Lefèvre, M.; Larouche, N.; Schmeisser, D.; Dodelet, J. P. J. Am. Chem. Soc. 2014, 136, 978.

[99] Choi, C. H.; Park, S. H.; Woo, S. I. Green Chem. 2011, 13, 406.

[100] Bao, X. G.; Deak, D.; Biddinger, E. J.; Ozkan, U. S.; Hadad, C. M. Chem. Commun. 2010, 46, 8621.

[101] Yang, L.; Jiang, S.; Zhao, Y.; Zhu, L.; Chen, S.; Wang, X.; Wu, Q.; Ma, J.; Ma, Y.; Hu, Z. Angew. Chem., Int. Ed. 2011, 50, 7132.

[102] Shui, J.-L.; Karan, N. K.; Balasubramanian, M.; Li, S.-Y.; Liu, D.-J. J. Am. Chem. Soc. 2012, 134, 16654.

[103] Chen, J.; Takanabe, K.; Ohnishi, R.; Lu, D.; Okada, S.; Hatasawa, H.; Morioka, H.; Antonietti, M.; Kubota, J.; Domen, K. Chem. Commun. 2010, 46, 7492.

[104] Qi, J.; Jiang, L.; Jiang, Q.; Wang, S.; Sun, G. J. Phys. Chem. C 2010, $114,18159$.

[105] Zhang, K.; Zhang, L.; Chen, X.; He, X.; Wang, X.; Dong, S.; Han, P.; Zhang, C.; Wang, S.; Gu, L.; Cui, G. J. Phys. Chem. C 2013, 117, 858.

[106] Li, F.; Ohnishi, R.; Yamada, Y.; Kubota, J.; Domen, K.; Yamada, A.; Zhou, H. Chem. Commun. 2013, 49, 1175. 